\title{
Train Tracking and the Signaling System using Infrared and Radio Frequency Technology
}

\author{
K. Vidyasagar \\ Dept. of ECE, SSIT \\ Sathupally, T.S, India
}

\author{
P.Sekhar Babu \\ Dept. of ECE, SSIT \\ Sathupally, T.S, India
}

\author{
R.Ram Prasad \\ Dept. of ECE, SSIT \\ Sathupally, T.S, India
}

\begin{abstract}
This paper considered the secured level crossing Signaling system and Train tracking system. This paper adopted a switching logic methodology to meet the challenges of the tracking system. This work also focused to map the train on the display screen. If the train moving close to the level crossing system the signaling system causes to release the green signal that causes to close the rail gate to avoid the unauthorized entry on the rail track. The open state of the gate is influenced by the signaling system by means of a 'Red'colour signal. The train is allowed to move on the track only by considering the green signal. The rail track is organized with four stop positions. Stop 1, stop 2, stop 3 and stop 4 . The locations of the train at various stop positions are sensed by using IR sensors. The detected signal is transmitted to the control room using RF transmitter operating at 433 MHZ. An Atmel micro controller is used to regulate the entire process to meet the desired state of the work. This proposed methodology had been successfully implemented on the 30 feet length of the scaled model of the rail track in the laboratory. The system results progressive response while tracking the position of the train. The results are recorded and analyzed. The proposed system may keep alert the monitoring mechanism. So, the collision of the train and unexpected human errors can be minimized.
\end{abstract}

\section{Keywords}

Tracking system, IR Sensors, RF Transmitter

\section{INTRODUCTION}

Indian railways daily transporting 14 million passengers by 2 million kilometer rail track per day. Safety is outstanding significance to Indian railways. Safety and reliability are closely linked components. Deterioration in the safety mechanism is preceded by increasing the number of failures. A man machine interface system inherently may enhance the reliability of the equipment. This is the most significant factor in the safety of the rail transport system. Collision avoidance mechanism, Secured level crossing system and signaling system having significance importance in railway system. Tracking the position of the train using Global position receiving system is proposed to be implemented in motor transport system. Indian railway system is looking forward to adopt the tracking methodology to have prior state of the train before arriving to the station. This component is having wide scope of research specifically for Indian rail system.

\section{LITERATURE SURVEY}

Md. Reya Shad Azim1 , Khizir Mahmud and C. K. Das proposed a rail track switching system for track controlling devices including railway switches and. A low power dc motor is used as a track switching device. Photodiode is used as a sensing device for detecting IR radiation which ensures a reliable detection of trains passage. This system can be operated both manually and automatically. Software is developed for this system for flexible control mechanism [1].

Anand.kr.Gupta et.al proposed A RFID based railway track system. The system implanted RFID Readers on the track and RFID tags at the bottom surface of the train for detection and transmission. This paper also considers determining the correct path of the train. The train is allowed to move only in correct path [2].

Shaik Nahid et.al proposed Rail tracking system (RTS).GPS is adopted for transmission and receiving of the signals. A communication link is provided between the RTS and control room using GPS. The location of the train is tracked using GSM technology [3].

Rajkumar et.al presents train tracking system using Global positioning system (GPS) and communication link provided using Ethernet concepts. A unique IP address provided to track the trains. This paper also focused to monitor the status of the alcohol dunked state of the driver and the speed of the train also. [4]

Niraj V et.al proposed RFID technology to regulate the entry pass of the train at the platform. RFID codes are provided to all the trains. The availability of the platform is communicated with the train using ZIG Bee technology.[5]

N. K. Das et.al adopted GPS -GPRS based train monitoring system. Track number is assigned to track the position of the train. The state of the train is linked to the web server to curtail the accidents. [6]

\section{THEORITICAL APPROACH}

\subsection{IR Sensor}

An Infrared (IR) sensor used to detect the motion of the train on the track. In the infrared spectrum all objects emits thermal radiation. That can be detected by infrared sensor. An IR Light emitting diode acts as an emitter and IR photo diode acts as a detector. The detector is sensitive to IR light. When IR signal falls on the photo diode the resistance is changed. The change of resistance is proportional to the voltage. This voltage is proportional to the magnitude of the received IR signal. The IR sensor at the transmitter transmits continuous IR rays. The output terminal of the IR receiver varies with respect to the received IR rays.

The total thirty feet length of scaled rail track is organized into four zones. Each Zone of the track represents one station. 
Four IR sensors are positioned at each station to detect the represents one station. Four IR sensors are positioned at each station to detect the location of the train. Two IR sensors are implanted before and after the level crossing gate for signaling purpose. These sensors will function in association with the state of open and close of the gate mechanism. The signal conversion is not considered since the output signal is an order of digital form. The output signal is interfaced to the encoder (HT- $12 \mathrm{E}$ ) for converting parallel data into serial data using AT89S52 microcontroller. The encoder interfaced to the microcontroller is shown in Fig 1.

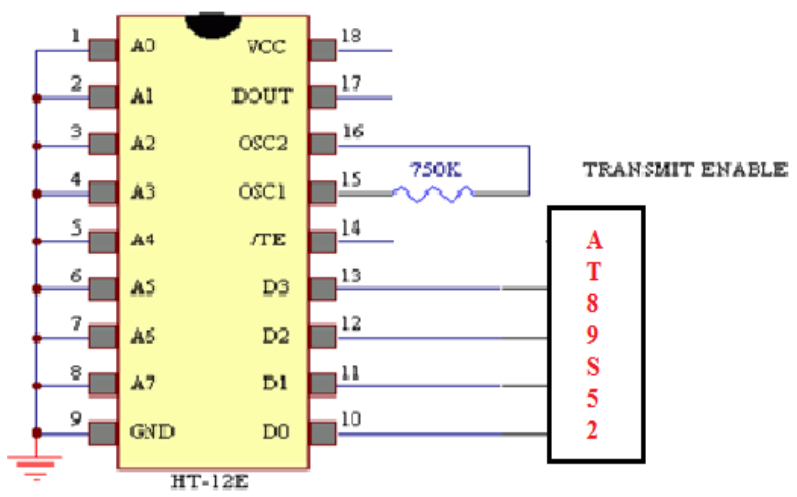

Fig 1: Simulated circuit of encoder interfaced to micro controller

\subsection{Communication}

Rf Transceiver operating at $433 \mathrm{MHz}$ is adopted as a communication device between the control room and the sensing stage of the circuit at the rail track. Since RF Communication works on serial communication an encoder is adopted in association with AT89S52 microcontroller to convert $\mathrm{n}$-bit into serial data. The encoder is TTL compatible. It is to be converted into serial data using encoder. The RF transmitter is used to read the serial data, and the signal is modulated using frequency shift keying method. The modulated signal is transmitted through the antenna. The RF receiver receives the modulated signal through the antenna. Filtering, processing and demodulation are to be done at the $\mathrm{RF}$ receiver. The serial data at the output stage of the RF Receiver is converted into TTL form. HT 12D-HT 12E pair is used as encoder and decoder.

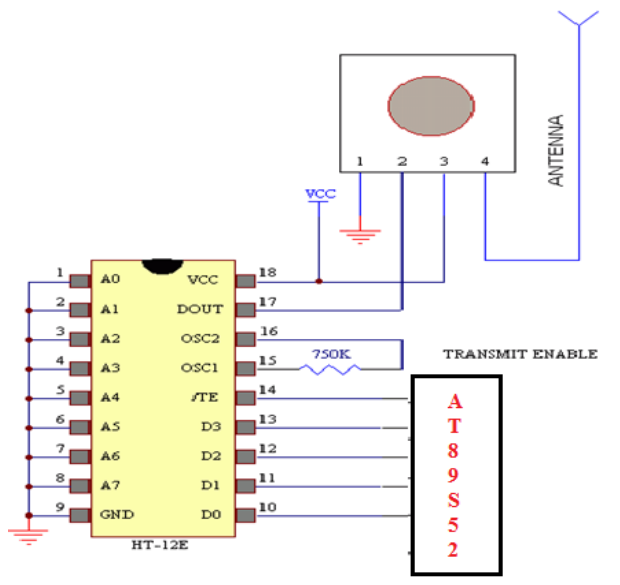

Fig 2: Simulated circuit of RF Transmitter interfaced to microcontroller

\subsection{Micro Controller}

AT89S52 microcontroller is used to track the position of the train. The received signal from the IR sensors is to be processed based on the logic developed. The processed signal is transmitted to the control room for tracking the state of the train. The micro controller receives input data from ' 6 ' no of IR sensors and RF transmitter section. It produce the processed output to the signaling system, which is whenever two level crossing gates closed signaling system green signal, otherwise shows red signal will be glows.

\section{IMPLEMENTED ALGORITHAM}

Step1. Initialize the port ' $0 \_0$ ', '0_1', '0_2', $0 \_3$ ' for IR sensor $1,2,3,4$, respectively

Step2. Equate IR1 for station 1, IR2 for station 2, IR3 for station 3 and IR4 for station 4.

Step3. Initialize port ' 044 ', ' 0 5' for I R Sensor 5 and 6

Step4. Equate IR5 for gate open state, IR6 for gate closed state.

Step5. Initialize port ' 06 ' for Buzzer

Step6. Initialize Port' 0_7 for Display device

Step7. Initialize port ' 3 - 0 ' for RF transmitter

Step8. Check the initial state '1111' for RED LED and '0000' for green LED of the display device

Step9. Read the IR sensor1 ( port 0_0 )

Step10. If the logic level is 5 volts Then

Enable the Interrupt- 4 to set $\mathrm{RI}=1$ and to transmit the status to the control room.

Output the logic state ' 1110 ' for RED LED and output '0001' for Green LED

Step11. Read IR 5 and IR 6 (port 0_4 and Port 0_5)

Step12. If the logic level is 5 volts

Then

Enable the green LED

Step 13. Read IR2

If logic level is 5 volts

Then

Enable the output port to transmit to the control room

Output the logic state ' 1100 ' for RED LED and output '0011'for Green LED

Else go to step 13.

Step14: Read IR 3

If logic level is 5 volts

Then

Enable the output port to transmit to the control room

Output the logic state '1000' for RED LED and output '0111'for Green LED

Else go to step 14.

Step15. Read IR4

If logic level is 5 volts Then

Enable the output port to transmit to the control room Output the logic state '0000' for RED LED and output '1111' for Green LED Else

Go to step 16 .

Step 16. Stop the process (Termination point)

The ports of the microcontroller both at the transmitted stage and at the receiving stage are initialized. The microcontroller reads the state of the IR sensors at each stage of the circuit. At the control room four Red LEDS and four Green LEDS are considered for tracking the position of the train. 


\section{RESULTS AND ANALYSIS}

The Experiments were conducted to produce the desired output tracking signals at the remote control room. The RF transmitter operating $433 \mathrm{MHz}$ frequency is used to transmit the status of the train to the control room.

Initially all the red LEDS are at the ON state i.e. "1111" in binary form and all the four Green LEDs are at OFF state i.e. "0000" in binary form.

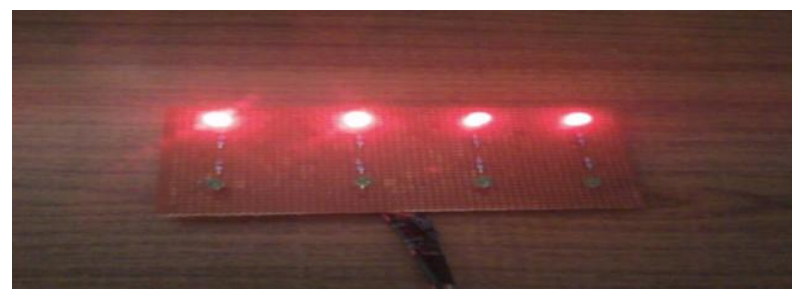

Fig 5.1: Initial state of the tracking system at the control room

The IR sensor located at the station 1 is detected causes to change the state of Red LEDs to "1110" and the state of green LEDS will be " 0001 ".

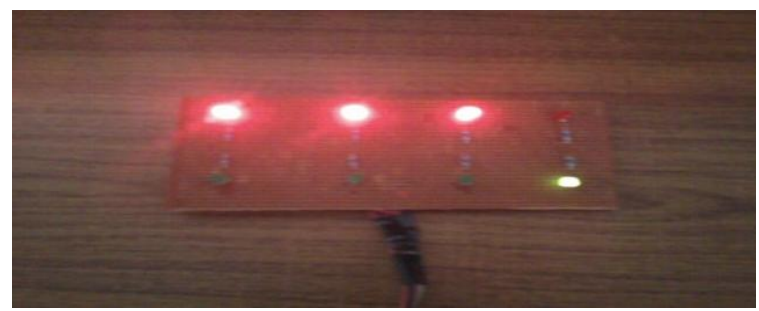

Fig 5.2: State of the tracking system at the control room when the train reached to station 1

The IR sensor at the level crossing gate is to be detected by the controller at the signal controlling circuit causes to indicate either green or red LED ON. Red LED on the track forced to stop the train. The green LED On state allows the train to move on the track. The controlling and RF transmitter circuit allowed detecting the IR 2 at the station 2. This detected signal causes to change the state of RED LED $s$ to "1100" and the green LEDS to be "0011" at the remote control room.

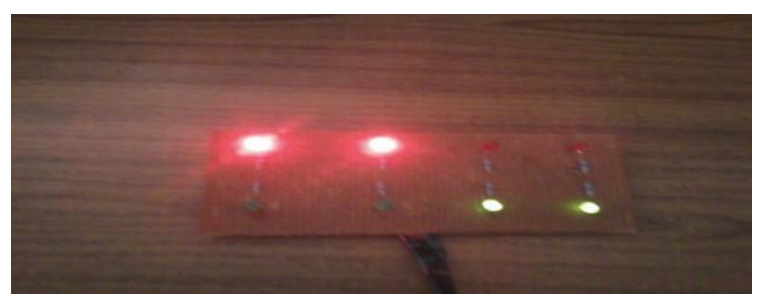

Fig 5.3: State of the tracking system at the control room when the train reached to station 2

When the train reaches to station 3 the RED LEDs state is represented with " 1000 " and the green LEDs state is represented with " 0111 ".

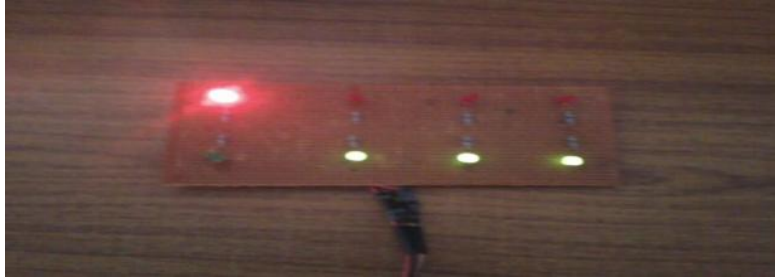

Fig 5.4: State of the tracking system at the control room when the train reached to station 3

When the train reaches to its destination i.e. at the station 4 the Red LEDs state is represented with " 0000 " and the green LEDs state is represented with " 1111 " in binary.

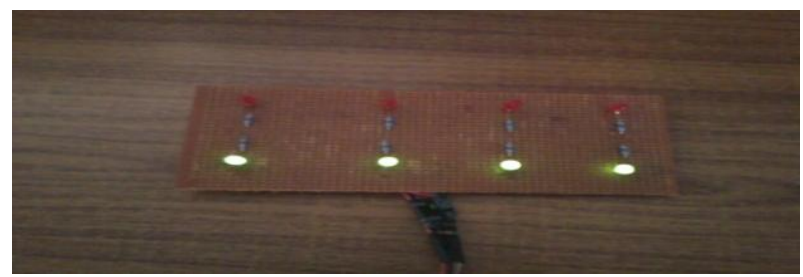

Fig 5.5: State of the tracking system at the control room when the train reached to station 4

The data at sensors will be transmitted to the control room by using RF transmitter module.The data received by the receiver send by the transmitter. The transmitter and receiver are in synchronous to each other with help of clock circuit. Receiving section at control room operates based on the receiving signal, that signal given to the level crossing gates. And they operate with the help of switching logic in the micro controller.

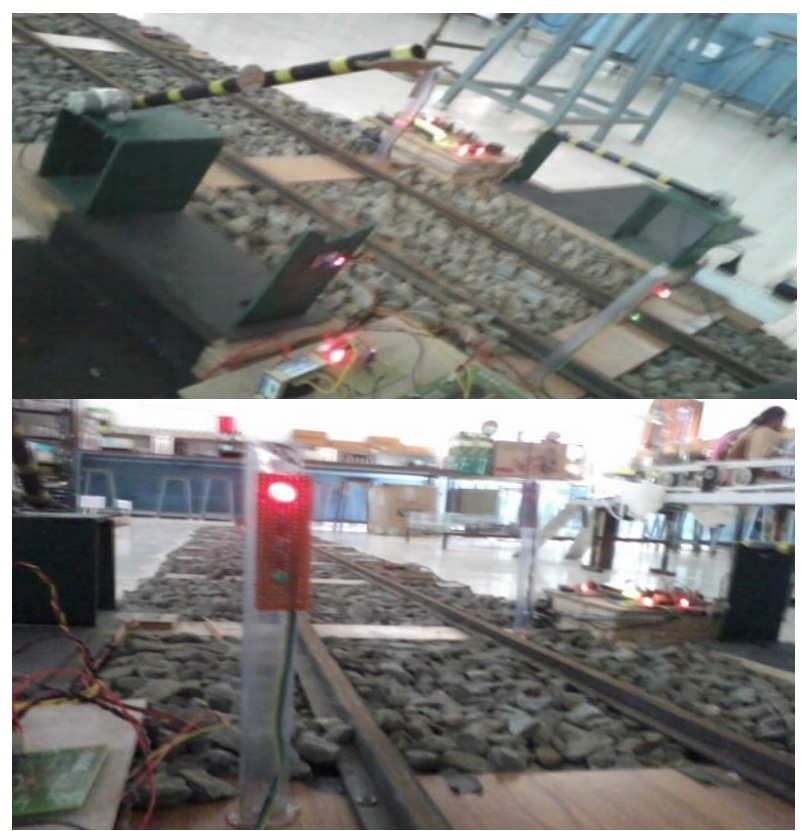

Fig 5.6: Signaling system of the gate open state indicates red signal on the track 


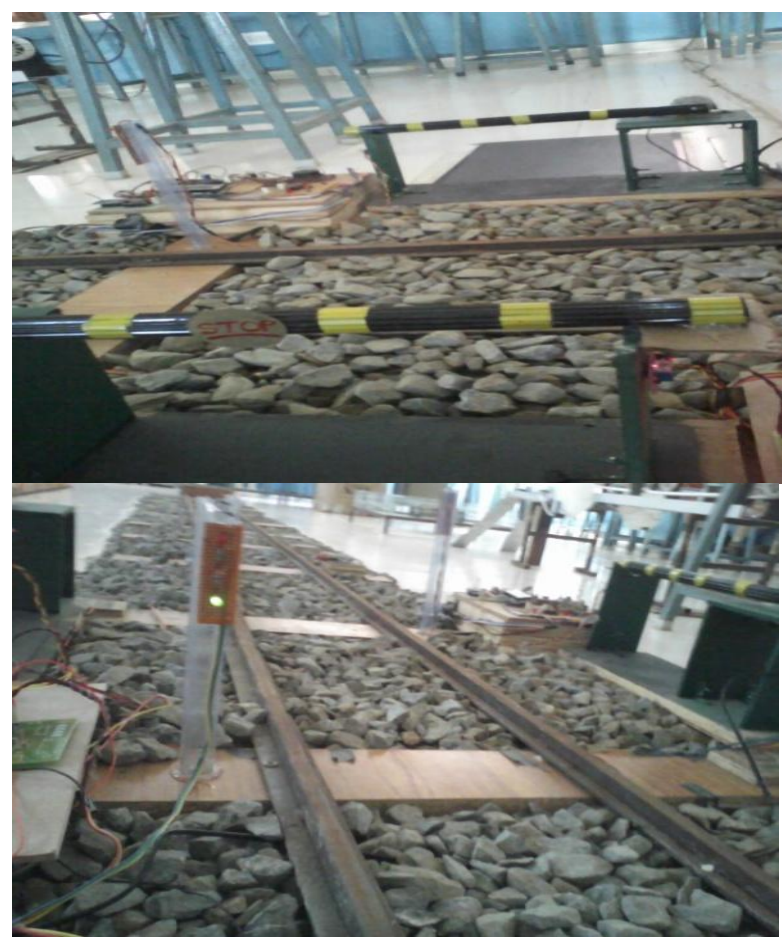

Fig 5.7: Signalling system of the gate closed state indicates green signal on the track

\section{CONCLUSION}

The proposed developed methodology produced reliable results. Tracking of the system using IR sensors and RF Technology may enable the rail department to safeguard the human life from accidents. The positional status of the train transmitted to the control room using RF technology can be replaced the manual human interpretation. Automating the monitoring system may curtail the human errors from train accidents.

In future rout mapping method with auto error correction mechanism may improve the safety mechanism to the rail transport system.

\section{ACKNOWLEDGMENTS}

The authors like to express sincere thanks to the management and principal of Sai Spurthi Institute of Technology for providing the fund and necessary infrastructure. And also thank to Mr. Prassanna Kumar in charge of Radio frequency department Sak informatics.

\section{REFERENCES}

[1] Md. Reya Shad Azim1 , Khizir Mahmud2 and C. K. Das. "Automatic Train Track Switching System with Computerized Control from the Central Monitoring Unit International Journal Vol 7(1) pages 201-214, 2014.

[2] Anand Kr.Gupta et.al, "Railway Track Finding System with RFID Application”, International Journal.

[3] Shaik Nahid, Srinivas Padala, V.Samson Deva Kumar, "Design and Development of Train Tracking System in South Central Railways", International Journal of Science and Modern Engineering, Voll (12),Pages 6064, Nov 2013.

[4] Rajkumar et.al , "GPS and Ethernet based real time train tracking system", Advanced Electronics Electronic Systems(ICAES) international conference, IEEE explore digital library, pages 282-286, 21-23 sept, 2013.

[5] Niraj V.et.al, "ZigBee and RFID Based Train Tracking System", International Journal of Thesis Projects and Dissertations, Vol. 2( 2), PP: (1-3), April - June 2014.

[6] N. K. Das. Et.al," Satellite Based Train Monitoring System", Journal of Electrical Engineering, Vol. EE 36(II), pages 35-38, December 2009. Science and Technology, Vol 7 (1), pages 201-212, 2014.

\section{AUTHOR BIOGRAPHY}

K.Vidyasagar: Received B. Tech degree in Instrument Technology from Andhra University College of Engineering Visakhapatnam, M.E from P.S.G.Tech Coimbatore. He is now a research scholar under the guidance of Dr. A. Bhujangarao, Andhra University. His current research interests include image processing in biomedical instrumentation and related embedded systems.

P. Sekhar Babu: Received B.Tech degree in ECE from AZCET, JNTU, and Hyderabad. M.TECH from V.R.S.E.C ANU, Guntur. He is now a research scholar of JNTU Kakinada. His research interests in wireless communication \& Signal Processing. He published his research work in several conferences.

R. Ram Prasad: Received his B.Tech degree in ECE from Sai Spurthi Institute of Technology, JNTU, and Hyderabad. M.Tech from Nova Engineering College, JNTU, and Kakinada. His research interest is on RF and Space Communication. He published several papers in various international conferences. 